\title{
DETERMINAÇÃO DE FÓSFORO BIODISPONÍVEL EM RAÇÕES DE PEIXES UTILIZANDO EXTRAÇÃO ASSISTIDA POR ULTRA-SOM E ESPECTROFOTOMETRIA NO VISÍVEL
}

\section{Paula M. de Moraes, Vanessa R. Loureiro e Pedro M. Padilha*}

Departamento de Química e Bioquímica, Instituto de Biociências, Universidade Estadual Paulista "Julio de Mesquita Filho", 18618-000 Botucatu - SP, Brasil

Renato de Cássio F. Neves, Mayra A. D. Saleh, Felipe A. dos Santos e Fábio A. Silva

Faculdade de Medicina Veterinária e Zootecnia, Universidade Estadual Paulista "Julio de Mesquita Filho", 18618-000 Botucatu - SP, Brasil

Recebido em 8/5/08; aceito em 18/11/08; publicado na web em 26/2/09

\begin{abstract}
DETERMINATION OF BIOAVAILABLE PHOSPHORUS IN FISH FEEDS BY ULTRASOUND EXTRACTION AND VISIBLE SPECTROPHOTOMETRY. The aim of the present work was to develop and optimize a method for determination of bioavailable phosphorus in samples of feces and fish feed using ultrasound extraction and subsequent quantification by visible spectrophotometry. Using as extractor solution $\mathrm{HNO}_{3} 0.50 \mathrm{~mol} \mathrm{~L}$, the great conditions of extraction established were: sample mass - $100 \mathrm{mg}$, samples granulometry - $<60 \mu \mathrm{m}$, sonification time - five cycles of $40 \mathrm{~s}$ and ultrasound potency - $136 \mathrm{~W}$. The proposed method was applied in studies of digestibility of this nutrient in different feeds used in diets of juvenile of Nile tilapia.
\end{abstract}

Keywords: ultrasound extraction; fish nutrition; phosphorus absorption.

\section{INTRODUÇÃO}

Os tecidos corporais dos animais possuem elementos químicos em proporções e quantidades variáveis. Neste contexto, os minerais são componentes essenciais ao metabolismo, refletindo na maior ou menor produtividade do animal. ${ }^{1}$ Dois a cinco por cento do corpo do animal é constituído pela fração mineral, que varia em função da espécie, da raça e do próprio indivíduo. ${ }^{2,3} \mathrm{O}$ fósforo é classificado como um macromineral, ou seja, mineral que o organismo necessita em grandes quantidades e, ainda, apresenta função estrutural, porque é parte integrante dos ossos, dentes e de algumas proteínas musculares. ${ }^{1-3}$

Os minerais podem interagir entre si, com outros nutrientes e também com alguns fatores não nutritivos da dieta. A interação do tipo sinérgica ou antagônica poderá ocorrer na própria dieta e durante o metabolismo no trato digestório. O conhecimento dessas interações assume importância fundamental para a correção das deficiências minerais causadas principalmente pelos desequilíbrios entre os elementos constituintes da dieta. No aspecto sinérgico, o fósforo pode ser citado como participante do processo intermediário da formação da parede intestinal, na atividade das enzimas digestivas e na formação dos ossos. ${ }^{4} \mathrm{Na}$ interação do tipo antagônica, em nível gastrintestinal, o fósforo pode inibir a absorção de outros elementos devido à formação de complexos como, por exemplo, ferro-fitatos e desencadear efeitos opostos sobre os ciclos bioquímicos no organismo.,

Em decorrência à sua essencialidade para o crescimento e para o metabolismo dos lipídios, o fósforo é o mineral de maior importância na nutrição animal. A maior quantidade de fósforo a ser metabolizado vem da dieta, enquanto que nos alimentos de origem vegetal este mineral se apresenta em grande parte na forma indisponível. Sua exigência na nutrição de peixes é dependente das características anatomofisiológicas do sistema digestório da espécie criada e da fonte desse mineral, sendo que na fonte alimentar, este elemento pode se apresentar na forma de fósforo orgânico como fitatos, fosfolipídios e

*e-mail: padilha@ibb.unesp.br fosfoproteínas e na forma inorgânica, como monofosfatos, bifosfatos e trifosfatos, solúveis ou não, no suco gástrico dos peixes..$^{7-10}$

A maior parte do fósforo presente nos ingredientes de origem vegetal, com destaque aos cereais, que são os principais componentes das formulações de rações, está na forma de fitato. Por isso, considera-se que apenas $30 \%$ do fósforo dos vegetais esteja disponível para não-ruminantes (monogástricos). ${ }^{7} \mathrm{~A}$ disponibilidade deve-se à quantidade de fósforo preso à molécula de ácido fítico ou simplesmente fitato. O fitato pode formar complexos do tipo quelato com íons metálicos como, por exemplo, ferro, cobre, zinco e manganês. O que além de deixar indisponível o fósforo e alguns nutrientes metálicos, interfere também na absorção de aminoácidos e pode inibir a atividade de várias enzimas digestivas endógenas, como tripsina, pepsina e amilase. ${ }^{7,11,12}$

Estudos da biodisponibilidade do fósforo na nutrição animal buscam interpretar dados que melhorem a absorção deste mineral em fontes alimentares que apresentem fósforo fítico. Com a melhor absorção desse nutriente, é possível eliminar efeitos negativos de fatores antinutricionais que resultam em crescimento reduzido, alta conversão alimentar, alterações hormonais e, em casos extremos, lesões nos órgãos. Além disso, favorece a diminuição da poluição ambiental devido à menor excreção fecal de fosfatos não absorvidos que permanecem na água de cultivo, cujo descarte é feito nos mananciais hídricos, o que contribui significativamente no processo de eutrofização do sistema aquático. ${ }^{13}$

Dessa forma, o desenvolvimento de novas metodologias que permitam a extração e a quantificação segura dos nutrientes inorgânicos, para posterior determinação do coeficiente de digestibilidade aparente desses nutrientes nos estudos de nutrição animal, torna-se fundamental. Neste contexto, a extração do analito por ultra-sonificação, apresenta-se como técnica bastante robusta. ${ }^{14-18}$ Os efeitos químicos provocados pelos ultra-sons ocorrem devido ao fenômeno da cavitação acústica, que é o processo de nucleação, crescimento e colapso de bolhas transientes em líquidos expostos a ondas ultra-sônicas de baixa frequiência $(<1 \mathrm{MHz})$. A energia liberada durante a cavitação acústica fornece excelentes perspectivas para o preparo e/ou trata- 
mento de amostras. ${ }^{15,18}$ No preparo de amostras, o colapso das microbolhas favorece a extração de espécies químicas a partir de materiais sólidos, bem como a dissolução destes ou completa mineralização no caso de compostos orgânicos. ${ }^{16-18}$ Assim, a utilização da extração de nutrientes metálicos de amostras biológicas por ultra-sonificação permite a eliminação da mineralização ácida que, pela metodologia clássica, se constitui no principal problema. ${ }^{19,20}$

Considerando o exposto, este trabalho descreve o desenvolvimento de um método para determinação de fósforo biodisponível de amostras de rações de peixes utilizando extração por ultra-som e quantificação por espectrofotometria no visível permitindo, assim, a estimativa do coeficiente de digestibilidade aparente desse nutriente em algumas fontes alimentares utilizadas na piscicultura.

\section{PARTE EXPERIMENTAL}

\section{Reagentes e soluções}

Água ultrapura (18,2 $\left.\mathrm{M} \Omega \mathrm{cm}^{-1}\right)$ obtida pelo sistema Elga modelo Purelab Ultra Ionic, ácido nítrico e clorídrico (Merck) de grau analítico foram utilizados no preparo das soluções extratoras. As soluções padrão de fósforo foram preparadas por diluições de padrão titrisol Merck e as soluções dos concomitantes foram preparadas a partir de reagentes de grau analítico, todos Merck. Todas as soluções foram estocadas em frascos de polipropileno.

Todos os frascos de estocagem de amostras e soluções padrão, vidrarias e as cubetas do espectrofotômetro foram lavados com ácido nítrico $10 \% \mathrm{v} / \mathrm{v}$ por $24 \mathrm{~h}$, em seguida, com água ultrapura e secos por jatos de ar puro antes da utilização.

\section{Coleta e preparo das amostras}

As amostras de fezes de peixes foram coletadas após experimentos de digestibilidade envolvendo juvenis de tilápia do Nilo que foram submetidos a uma dieta com diferentes fontes alimentares. Esses experimentos foram desenvolvidos no Laboratório de Nutrição de Peixes da Faculdade de Medicina Veterinária e Zootecnia/UNESP, Campus de Botucatu.

Depois de coletadas, as amostras de rações de peixes foram desidratadas em estufa de recirculação forçada de ar por $48 \mathrm{~h}$ e submetidas à moagem criogênica. Para isso, uma massa de aproximadamente $1,0 \mathrm{~g}$ da amostra foi colocada em frasco de policarbonato juntamente com a barra magnética, o qual devidamente fechado foi imerso em nitrogênio líquido. Pelo impacto entre a amostra e a barra magnética submetida a um campo magnético oscilante (20 impactos $\mathrm{s}^{-1}$ ) a amostra foi pulverizada. O programa utilizado na moagem das amostras compreendeu uma primeira etapa de 2 min para o pré-congelamento, 1 min de pulverização, novamente $1 \mathrm{~min}$ de congelamento e uma segunda etapa que compreendeu dois ciclos com dois estágios de pulverização e congelamento, perfazendo um tempo total de 8 min. Este procedimento permitiu obter partículas com granulometria menor que $60 \mu \mathrm{m} .^{21}$

\section{Instrumentação}

Estufa de circulação forçada de ar Tecnal modelo TE - 394/1.

A moagem das amostras foi feita em moinho criogênico SPEX - Freezer, modelo Mill 6750.

Desrruptor de células ultra-sônico Unique equipado com sonda de titânio de $3 \mathrm{~mm}$ foi utilizado no processo de extração do fósforo nas amostras de rações e fezes de peixes.

Forno de microondas Provecto Analítica (Campinas-SP) modelo DGT 100 plus foi utilizado para mineralização das amostras.
A determinação de fósforo foi feita utilizando espectrofotômetro Genesys $^{\mathrm{TM}}$ modelo 6.

\section{Extração do fósforo}

Após a etapa de secagem e moagem, aproximadamente 100 mg de amostra e $20 \mathrm{~mL}$ de solução de ácido clorídrico e/ou nítrico foram transferidas para frascos de teflon de $50 \mathrm{~mL}$. A mistura amostra/ solução foi então submetida à agitação por ultra-som para extração do fósforo. Adotando este procedimento foram avaliadas diferentes concentrações das soluções ácidas e diferentes tempos de sonificação e potências do ultra-som no processo de extração do analito. Os extratos ácidos obtidos foram separados da fase sólida remanescente por centrifugação, para posterior determinação da concentração de fósforo.

Outra parte das amostras foi também mineralizada em forno de microondas. Para isso, massas de 100 mg de amostras moídas criogenicamente foram transferidas diretamente para os frascos de teflon do forno de microondas, adicionando-se em seguida 2,50 $\mathrm{mL}$ de ácido nítrico $14 \mathrm{~mol} \mathrm{~L}^{-1}, 0,50 \mathrm{~mL}$ de ácido perclórico $12 \mathrm{~mol} \mathrm{~L}^{-1}$ e 0,50 mL de peróxido de hidrogênio $30 \% \mathrm{~m} / \mathrm{m}$. O programa de aquecimento utilizado foi o descrito no manual do fabricante do equipamento com algumas modificações. ${ }^{19,20}$ Este procedimento foi utilizado também na digestão dos extratos ácidos obtidos no processo de extração por ultra-som, utilizando-se no caso volumes de 3,00 $\mathrm{mL}$ dos extratos, 1,50 $\mathrm{mL}$ de ácido nítrico concentrado, $0,50 \mathrm{~mL}$ de ácido perclórico $12 \mathrm{~mol} \mathrm{~L}^{-1}$ e $0,50 \mathrm{~mL}$ de peróxido de hidrogênio $30 \% \mathrm{~m} / \mathrm{m}$.

\section{Determinação de fósforo nas amostras de ração e fezes de peixes}

A determinação de fósforo foi feita utilizando o método espectrofotométrico do ácido vanadomolibdicofosfórico. Esse método fundamenta-se na reação de íons ortofosfato, em meio ácido, com íons molibdato e vanadato com conseqüente formação de um heteropoliácido complexo de cor amarela. Tal complexo absorve radiação visível em $420 \mathrm{~nm}$, comprimento de onda correspondente à cor violeta, complementar da cor verde amarelado. A intensidade de radiação absorvida pelo heteropoliácido complexo é proporcional à quantidade de ortofosfato $\left(\mathrm{H}_{2} \mathrm{PO}_{4}^{2-}\right)$ e/ou fósforo presente nos extratos ácidos. ${ }^{22}$

\section{RESULTADOS E DISCUSSÃO}

\section{Otimização do tempo e da potência de sonificação no processo de extração dos analitos metálicos}

A eficiência do processo de extração por ultra-som de fósforo das amostras de rações e fezes foi avaliada variando as concentrações das soluções ácidas extratoras, o tempo de extração e a potência ultrasônica. $\mathrm{Na}$ etapa de moagem da amostra procurou-se obter a menor granulometria possível, o que favorece a homogeneidade do analito no material sólido e evita possíveis erros associados à representatividade da amostra. Nessa etapa também foi evitada a manipulação excessiva das amostras procurando, assim, evitar possíveis fontes de contaminação. A quantidade de material sólido pesado (100 mg) foi feita considerando a concentração do nutriente na formulação da ração. O efeito da concentração do ácido clorídrico e nítrico no processo de extração do fósforo foi avaliado na faixa de 0,10 a 1,00 mol $\mathrm{L}^{-1}$, medindo o sinal de absorbância nos extratos ácidos obtidos com cada uma dessas soluções, após a reação com o agente cromogênico. Observou-se que o maior sinal de absorbância foi obtido utilizando ácido nítrico $0,50 \mathrm{~mol} \mathrm{~L}^{-1}$ (Figura 1). Dessa forma, essa solução extratora foi utilizada em todos os demais experimentos. 


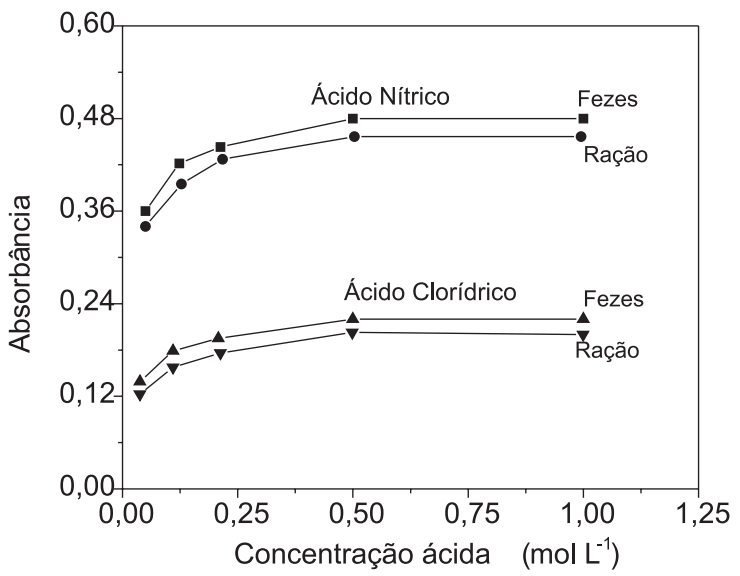

Figura 1. Influência da concentração das soluções de ácido clorídrico e nítrico no processo de extração de fósforo de amostras de rações e fezes de peixes. Condições experimentais: tempo de sonificação - 5 ciclos de $40 \mathrm{~s}$; potência utilizada $-136 \mathrm{~W}$

Conforme já discutido, as ondas ultra-sônicas promovem a extração dos analitos da matriz devido à atuação dessas sobre as moléculas orgânicas presentes na amostra, destruindo-as e provocando a quebra da ligação do analito com essas moléculas. ${ }^{14-18}$ Assim, foram feitos experimentos de otimização do tempo e da potência de sonificação no processo de extração do fósforo das amostras de rações e fezes, utilizando ácido nítrico $0,50 \mathrm{~mol} \mathrm{~L}^{-1}$ como solução extratora. As Figuras 2 e 3 mostram os resultados obtidos nesses experimentos.

$\mathrm{O}$ aumento do tempo de sonificação provoca o aumento da temperatura do meio extrator. Normalmente, observa-se que numa temperatura em torno de $50{ }^{\circ} \mathrm{C}$, a eficiência de extração é favorecida devido ao aumento do número de núcleos de cavitação acústica formada nas proximidades da superfície da fase sólida da suspensão (sistema sólido-líquido). ${ }^{23}$ No entanto, quando a temperatura do meio extrator se aproxima da temperatura de ebulição da fase líquida, ocorre um decréscimo na eficiência da sonificação devido à diminuição da tensão superficial do meio e aumento da pressão de vapor dentro das microbolhas, o que provoca uma diminuição nas ondas de choque..$^{24}$ Existe, dessa forma, um tempo considerado ótimo de sonificação no processo de extração do analito de uma amostra sólida. Nesse tempo ocorre a cavitação acústica ou o ciclo de formação, crescimento e colapso das microbolhas formadas pela

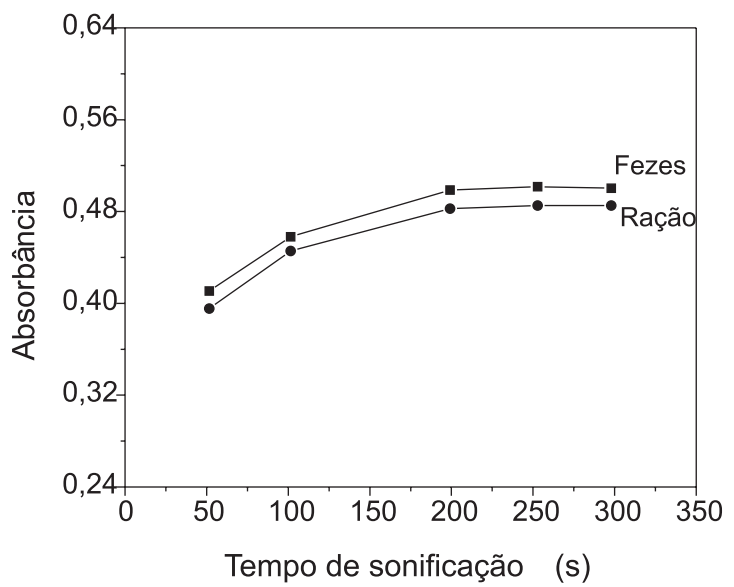

Figura 2. Influência do tempo de sonificação no processo de extração de fósforo de amostras de rações e fezes de peixes. Condições experimentais: tempo de sonificação - 5 ciclos de 10, 20, 40, 50 e 60 s; potência utilizada$136 \mathrm{~W}$; solução extratora - ácido nítrico $0,50 \mathrm{~mol} \mathrm{~L}^{-1}$

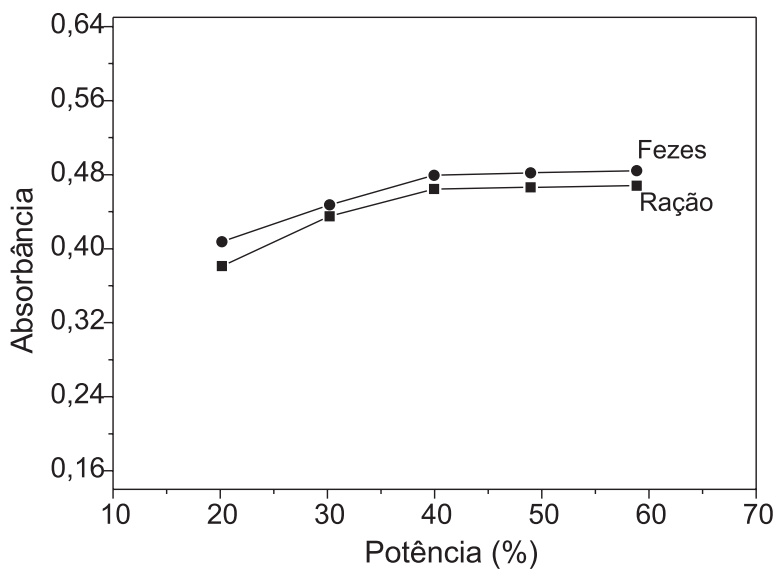

Figura 3. Influência da potência de sonificação no processo de extração de fósforo de amostras de rações e fezes de peixes. Condições experimentais: tempo de sonificação - 5 ciclos de 40 s; potência utilizada - 20, 30, 40, 50 e $60 \%$ da potência total do equipamento $(340 \mathrm{~W})$; solução extratora - ácido nítrico $0,50 \mathrm{~mol} \mathrm{~L}^{-1}$

propagação das ondas. Após o colapso das bolhas de cavitação, ocorre a liberação de grande quantidade de energia na microrregião próxima da superfície da fase sólida provocando, assim, a extração do analito e, em alguns casos, até a dissolução do material sólido. ${ }^{25}$ Analisando os gráficos apresentados na Figura 2, observa-se que a partir de 5 ciclos de $40 \mathrm{~s}$ de agitação ultra-sônica (200 s no total), os sinais de absorbância medidos permaneceram praticamente constantes, sendo que a temperatura medida no sistema sólido-líquido foi de $66^{\circ} \mathrm{C}$. Dessa forma, este tempo foi considerado eficiente no processo de extração dos analitos metálicos.

A intensidade do ultra-som transmitida ao meio está diretamente relacionada à amplitude da vibração da ponta do sonotrodo. Geralmente, um aumento na intensidade da potência favorecerá um aumento nos efeitos químicos provocados pela sonificação. Quando se utiliza amplitude vibracional muito elevada, um grande número de bolhas de cavitação são geradas na solução, o que pode desfavorecer o crescimento e o colapso dessas bolhas, tendo como conseqüência uma diminuição da energia liberada através da fase líquida. ${ }^{17,18,24}$ A eficiência da extração do fósforo, conforme mostra a Figura 3, aumenta com amplitude crescente de 20 a 30\% (68 a $102 \mathrm{~W}$ ), permanecendo constante para os valores mais elevados de amplitude. Os resultados obtidos indicaram que a eficiência máxima do processo de extração foi conseguida na escala de amplitude 40 a 60\% (136 a 204 W) de potência. Optou-se então por utilizar a amplitude de $136 \mathrm{~W}$ de potência em todos os demais experimentos, pelo fato dessa potência provocar um menor aumento de temperatura da solução extratora (66 ${ }^{\circ} \mathrm{C}$ ) contra, respectivamente, 74 e $82{ }^{\circ} \mathrm{C}$ para as amplitudes de 170 e $204 \mathrm{~W}$ de potência.

\section{Figuras de mérito do método de extração proposto}

Após a otimização dos parâmetros físico-químicos (concentração da solução extratora, tempo e potência de agitação ultra-sônica), a exatidão e a precisão do método de extração foram testadas utilizando ensaios de recuperação de fósforo de amostras de rações e fezes de peixes e também em uma amostra de padrão de ração certificado, cujos teores desses nutrientes foram determinados previamente utilizando a mineralização ácida em forno de microondas. Os resultados dessas determinações são mostrados na Tabela 1.

Analisando os resultados obtidos utilizando a extração por ultra-som e a mineralização das amostras (Tabela 1), observa-se que em ambas as metodologias os desvios-padrão relativos calculados foram menores que 
Tabela 1. Concentração de fósforo em amostras de rações e fezes de peixes, utilizando extração por ultra-som e mineralização em forno de microondas $(\mathrm{n}=5)$

\begin{tabular}{lccc}
\hline $\begin{array}{l}\text { Amostras de } \\
\text { rações e fezes }\end{array}$ & $\begin{array}{c}\text { Extração por } \\
\text { sonificação } \\
\left(\mathrm{mg} \mathrm{kg}^{-1}\right)\end{array}$ & $\begin{array}{c}\text { Extração por } \\
\text { mineralização } \\
\left(\mathrm{mg} \mathrm{kg}^{-1}\right)\end{array}$ & $\begin{array}{c}\text { Extrato ultra- } \\
\text { sônico digerido } \\
\left(\mathrm{mg} \mathrm{kg}^{-1}\right)\end{array}$ \\
\hline Ração $_{1}$ & $992 \pm 21$ & $1091 \pm 25$ & $1026 \pm 14$ \\
Ração $_{2}$ & $997 \pm 23$ & $1095 \pm 28$ & $1032 \pm 12$ \\
Fezes $_{1}$ & $1345 \pm 31$ & $1896 \pm 42$ & $1792 \pm 21$ \\
Fezes $_{2}$ & $1482 \pm 35$ & $2062 \pm 51$ & $1945 \pm 25$ \\
Ração $_{\text {Certificada* }}$ & $170 \pm 2,12$ & $172 \pm 2,21$ & $170 \pm 3,60$ \\
\hline
\end{tabular}

*Corn Bran, RM 8433 - National Institute of Standards and Technol$o g y$, valor certificado $=171 \pm 11,00 \mathrm{mg} \mathrm{kg}^{-1}$

$3 \%$, mostrando que estas apresentaram boa precisão (repetibilidade entre as medidas). No entanto, os resultados obtidos utilizando a extração por ultra-som apresentaram valores cerca de $10 \%$ menores para as amostras de ração e cerca de $40 \%$ menores para as amostras de fezes, quando comparados com as porcentagens de fósforo determinadas utilizando a mineralização ácida no processo de extração. Essas diferenças possivelmente estão relacionadas ao fósforo fítico presente nas amostras de rações e fezes, que no processo de ultra-sonificação não é extraído na forma de di-hidrogenofosfato para a reação com os íons vanadato e molibdato, ${ }^{22}$ reação utilizada na determinação do analito conforme descrito anteriormente. Para confirmar essa hipótese foram feitas determinações de fósforo nos extratos obtidos da extração por ultra-som procedendo-se à digestão ácida desses em forno de microondas. Os resultados obtidos (Tabela 1) indicam que, após a digestão em forno de microondas dos extratos obtidos por sonificação, houve um aumento nas concentrações de fósforo, que apresentaram valores cerca de $6 \%$ menores em relação às concentrações determinadas nos extratos obtidos por digestão direta das amostras, o que reforça a hipótese da fração de fósforo fítico não ser mineralizada no processo de extração por ultra-som. Em relação aos resultados determinados para a ração certificada, mostrados na Tabela 1, observa-se que além dos valores apresentarem desvios-padrão relativos menores que $2 \%$, o que caracteriza uma boa repetibilidade entre os resultados, também estão bem próximos dos valores certificados, comprovando assim a exatidão do método de extração proposto.

\section{Aplicação do método}

O método de extração proposto foi aplicado em determinações de rotina de fósforo, para posterior cálculo dos coeficientes de digestibilidade desse nutriente em quatro fontes alimentares utilizadas na dieta de juvenis de tilápia do Nilo. Os resultados dessas determinações foram comparados com os resultados obtidos utilizando a mineralização ácida das amostras em forno de microondas (Tabelas 2 e 3 ).

Com base nos valores das porcentagens de fósforo determinados nas amostras de rações e fezes da espécie em estudo, após a conversão dos dados apresentados nas Tabelas 2 e 3 e na determinação da porcentagem de óxido de crômio (marcador biológico utilizado em estudos de digestibilidade de nutrientes na nutrição animal, determinado conforme procedimento descrito por Silva et al.), ${ }^{19}$ foram calculados os coeficientes de digestibilidade aparente $(D a)$ do fósforo na dieta, a qual foi fornecida a juvenis de tilápia do Nilo, utilizando a Equação 1.26-28

$$
\mathrm{Da}=100-\left[100\left(\frac{\% \mathrm{Cr}_{2} \mathrm{O}_{3} \mathrm{r}}{\% \mathrm{Cr}_{2} \mathrm{O}_{3} \mathrm{f}}\right) \times\left(\frac{\% \mathrm{Nf}}{\% \mathrm{Nr}}\right)\right]
$$

Tabela 2. Concentração de fósforo em amostras de rações preparadas a partir de quatro fontes alimentares utilizadas na dieta de juvenis de tilápia do Nilo $(n=5)$

\begin{tabular}{|c|c|c|}
\hline \multirow{2}{*}{ Amostras de rações } & \multicolumn{2}{|c|}{ Concentração de Fósforo $\left(\mathrm{mg} \mathrm{kg}^{-1}\right)$} \\
\hline & Sonificado & Mineralizado \\
\hline Ração $_{1}$ & $1162 \pm 23$ & $1289 \pm 25$ \\
\hline $\mathrm{Ração}_{2}$ & $1140 \pm 21$ & $1260 \pm 26$ \\
\hline $\mathrm{Ração}_{3}$ & $1152 \pm 20$ & $1267 \pm 27$ \\
\hline Ração $_{4}$ & $1090 \pm 19$ & $1210 \pm 24$ \\
\hline
\end{tabular}

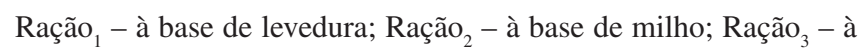
base de farelo de soja; Ração $_{4}$ - à base de farelo de arroz

Tabela 3. Concentração de fósforo em amostras de fezes de juvenis de tilápia do Nilo $(\mathrm{n}=5)$

\begin{tabular}{ccc}
\hline Amostras de fezes & $\begin{array}{c}\text { Concentração de Fósforo }\left(\mathrm{mg} \mathrm{kg}^{-1}\right) \\
\text { Sonificado }\end{array}$ & Mineralizado \\
\hline Amostra $_{1}$ & $1417 \pm 29$ & $2568 \pm 52$ \\
Amostra $_{2}$ & $1400 \pm 27$ & $2450 \pm 49$ \\
Amostra $_{3}$ & $1405 \pm 25$ & $2459 \pm 51$ \\
Amostra $_{4}$ & $1370 \pm 23$ & $2500 \pm 46$ \\
\hline
\end{tabular}

Amostra $_{1}$ - fezes de peixes alimentados com a Ração ; Amostra $_{2}$ fezes de peixes alimentados com a Ração; Amostra $_{3}$ - fezes de peixes alimentados com a Ração ${ }_{3}$; Amostra $_{4}$ - fezes de peixes alimentados com a Ração

onde, $D a=$ coeficiente de digestibilidade aparente; $\% \mathrm{Cr}_{2} \mathrm{O}_{3} \mathrm{r}=$ porcentagem de óxido de crômio na ração; $\% \mathrm{Cr}_{2} \mathrm{O}_{3} \mathrm{f}=$ porcentagem de óxido de crômio nas fezes; $\% \mathrm{Nr}=$ porcentagem do nutriente na ração e $\% \mathrm{Nf}=$ porcentagem do nutriente nas fezes.

Os valores dos $D a$, calculados a partir dos resultados das determinações de fósforo utilizando o método de extração por ultra-som e a mineralização das amostras em forno de microondas, estão sumarizados na Tabela 4.

Comparando-se os valores dos $D a$ do fósforo presentes nas quatro rações utilizadas na dieta de juvenis de tilápia do Nilo apresentados na Tabela 4, observa-se que os $D a$ obtidos, utilizando o teor de fósforo total (amostras mineralizadas), apresentaram valores entre $24-27 \%$ menores que os obtidos considerando as porcentagens de fósforo determinadas utilizando ultra-som no processo de extração do analito. As rações utilizadas na dieta dos peixes foram preparadas a partir de fontes alimentares de origem vegetal; essas fontes alimentares apresentam de 50 a $95 \%$ do teor total de fósforo na forma de ácido fitico (hexafosfato de inositol). Os peixes não apresentam a enzima fitase endógena que promove a hidrólise da ligação éster entre o fosfato e a molécula de inositol, disponibilizando assim o fósforo presente na

Tabela 4. Coeficiente de digestibilidade aparente do fósforo das quatro rações utilizadas na dieta de juvenis de tilápia do Nilo

\begin{tabular}{lcc}
\hline $\begin{array}{l}\text { Amostras de } \\
\text { rações }\end{array}$ & $\begin{array}{c}\text { Coeficiente de Digestibilidade Aparente - Da (\%) } \\
\text { Sonificado }\end{array}$ & Mineralizado \\
\hline Ração $_{1}$ & $58,54 \pm 1,20$ & $32,26 \pm 0,65$ \\
Ração $_{2}$ & $58,24 \pm 1,12$ & $33,88 \pm 0,71$ \\
Ração $_{3}$ & $58,53 \pm 1,04$ & $34,01 \pm 0,71$ \\
Ração $_{4}$ & $57,27 \pm 0,96$ & $29,75 \pm 0,55$ \\
\hline
\end{tabular}


molécula do ácido fítico. ${ }^{26,29-32}$ Como o método de extração por ultrasom não mineraliza a fração fítica, os $D a$ calculados utilizando esse método de extração são maiores porque na relação $\% \mathrm{~N}_{\mathrm{f}} / \% \mathrm{~N}_{\mathrm{r}}$ foram computadas somente as frações de fósforo disponível nas rações, ou seja, as frações extraídas na forma de di-hidrogefosfato. Dessa forma, pode-se inferir que a extração por ultra-som possibilita a obtenção de resultados de digestibilidade de fósforo mais coerentes com a necessidade de absorção deste nutriente pela espécie tilápia do Nilo, considerando que esses são concordantes com os resultados obtidos por pesquisadores que utilizaram a suplementação da enzima fitase em alimentos vegetais para disponibilizar o fósforo fítico. ${ }^{33-35}$

\section{CONCLUSÕES}

O método proposto de extração de fósforo por ultra-som permitiu calcular os $D a$ desse nutriente em quatro amostras de rações utilizadas na dieta de juvenis de tilápia do Nilo. Como nesse processo de extração não ocorre a mineralização da fração de fósforo na forma de fitato, é possível obter o teor biodisponível desse nutriente, o que permite a estimativa de $D a$ que caracteriza a absorção do fósforo da dieta a qual os peixes foram submetidos. De forma geral, deve-se destacar que a metodologia proposta diminuiu consideravelmente o tempo de análise, o que favoreceu a velocidade analítica. Outro fato a ser destacado é que no método de extração por ultra-som o óxido crômico que é adicionado nas rações como indicador biológico inerte ${ }^{19,20}$ não é solubilizado e, conseqüientemente, o crômio(III) não é oxidado a dicromato, espécie química extremamente tóxica, formada no processo de mineralização nítrica/perclórica das amostras.

\section{AGRADECIMENTOS}

À FAPESP (Processos 03/13362-6 e 06/51556-5) e ao CNPq (Processos 474371/2007-7 e 301123/2005-5) pelo suporte financeiro.

\section{REFERÊNCIAS}

1. McDowell, L. R.; Minerals in Animal and Human Nutrition, Academic Press: London, 1992.

2. Solomons, N. W.; Absorption and Malabsorption of Mineral Nutrients, Alan R. Liss: New York, 1992.

3. Smith, R. M.; Trace Elements in Human and Animal Nutrition, Academic Press: New York, 1987.

4. Tacon, A. G. J.; Standard Methods for the Nutrition and Feeding of Farmed Fish and Shrimp. The Essential Nutrients, Argent Laboratories Press: Washington, 1990, vol. 1.

5. Hilton, J. W.; Aquaculture 1989, 79, 223.

6. Lovell, T.; Nutrition and Feeding of Fish, Van Nostrand Reinhold: New York, 1989.

7. NRC (National Research Council); Nutrient Requirements of Fish, National Academy Press: Washington DC, 1993.

8. Steffens, W.; Principios Fundamentales de la Alimentación de los Peces, Acribia: Zaragoza, 1987.
9. Vielma, J.; Lall, S. P.; Koskela, J.; Schoner, F.; Mattila, P.; Aquaculture 1998, 163, 309.

10. Pizzolante, C. C.; Tese de Doutorado, Universidade Federal de Lavras, Brasil, 2000.

11. Kies, A. K. Em Phytase in Animal Nutrition and Waste Management; Coelho, M. B.; Kornegay, E. T., eds.; BASF Corporation: New Jersey, 1996.

12. Selle, P. H.; The Potential of Microbial Phytase for the Sustainable Production of Pigs and Poultry: an Australian Perspective, Sevent Short Course on Feed Techonology, Korean Society of Animal Nutrition and Feedstuffs: Ansung, 1997.

13. Gonçalves, G. S.; Pezzato, L. E.; Barros, M. M.; Hisano, H.; Freire, E. S.; Ferrari, J. E. C.; Acta Scientarium 2004, 26, 313.

14. Mason, T. J.; Lorimer, J. P.; Sonochemistry: Theory, Applications and Uses of Ultrasound in Chemistry, Ellis Horwood: New York, 1988.

15. Francony, A.; Pétrier, C.; Ultrason. Sonochem. 1996, 3, S77.

16. Nascentes, C. C.; Korn, M.; Arruda, M. A. Z.; Microchem. J. 2001, 69, 37.

17. Ruiz-Jiménez, J.; Luque-Garcia, J. L.; Castro, M. D. L.; Anal. Chim. Acta 2003, 480, 231.

18. Korn, M.; Pereira, M. G.; Borges, S. S.; Boletim da Sociedade Portuguesa de Química 2005, 96, 51.

19. Silva, F. A.; Padilha, C. C. F.; Pezzato, L. E.; Barros, M. M.; Padilha, P. M.; Talanta 2006, 69, 1025.

20. Silva, F. A.; Neves, R. C. F.; Quintero-Pinto, L. G.; Padilha, C. C. F.; Jorge, S. M. A.; Barros, M. M.; Pezzato, L. E.; Padilha, P. M.; Chemosphere 2007, 68, 1542.

21. Rosa, C. R.; Moraes, M.; Neto, J. A. G.; Nóbrega, J. A.; Nogueira, A. R. A.; Food Chem. 2002, 79, 517.

22. Willians, W. J.; Handbook of Anion Determination, Butterworths: London, 1979 .

23. Filgueiras, A. V.; Capelo, J. L.; Lavilla, I.; Bendicho, C.; Talanta 2000, $53,433$.

24. Elik, A.; Talanta 2005, 66, 882.

25. Elik, A.; Talanta 2007, 71, 790 .

26. Furuya, W. M.; Pezzato, L. E.; Pezzato, A. C.; Barros, M. M.; Miranda, E. C.; Rev. Bras. Zootecn. 2001, 30, 1143.

27. Pezzato, L. E.; Miranda, E. C.; Barros, M. M.; Quintero-Pinto, L. G.; Furuya, W. M.; Pezzato, A. C.; Rev. Bras. Zootec. 2002, 31, 1595.

28. Sá, M. V. C.; Pezzato, L. E.; Barros, M. M.; Padilha, P. M.; Aquaculture 2004, 238, 385.

29. Nolan, K. B.; Dufafin, P. A.; J. Sci. Food Agric. 1987, 40, 79.

30. Rodehutscord, M.; Pfeffer, E.; Aquaculture Magazine 1996, 1, 80.

31. Shafer, A.; Koppe, W. M.; Meyer-Burgdorff, H. G.; Water Sci. Technol. 1995, 31, 140 .

32. Oliva-Teles, A.; Pereira, J. P.; Gouveia, A.; Aquatic Living Resource 1998, 11, 255 .

33. Vielma, J.; Lall, S. P.; Koskela, J.; Aquaculture 1998, 63, 309.

34. Foster, I.; Higgs, D. A.; Dosanjh, B. S.; Aquaculture Nutrition 1999, 179, 109.

35. Gonçalves, G. S.; Pezzato, L. E.; Padilha, P. M.; Barros, M. M.; Rev. Bras. Zootec. 2007, 36, 1473. 